\title{
Dietary combination of sucrose and linoleic acid causes skeletal muscle metabolic abnormalities in Zucker fatty rats through specific modification of fatty acid composition
}

\author{
Hirokazu Ohminami, ${ }^{1, \uparrow}$ Kikuko Amo, ${ }^{1,+}$ Yutaka Taketani, ${ }^{1, *}$ Kazusa Sato, ${ }^{1}$ Makiko Fukaya, ${ }^{1}$ Takashi Uebanso, ${ }^{1}$ \\ Hidekazu Arai, ${ }^{1,2}$ Megumi Koganei, ${ }^{3}$ Hajime Sasaki, ${ }^{3}$ Hisami Yamanaka-Okumura, ${ }^{1}$ Hironori Yamamoto ${ }^{1}$ and Eiji Takeda ${ }^{1}$ \\ 'Department of Clinical Nutrition, Institute of Health Biosciences, The University of Tokushima Graduate School, 3-18-15 Kuramoto, Tokushima 770-8503, Japan \\ ${ }^{2}$ Department of Laboratory of Clinical Nutrition Management, School of Food and Nutritional Sciences \& Graduate School of Integrated Pharmaceutical and \\ Nutritional Sciences, The University of Shizuoka, 52-1 Yada, Suruga-ku, Shizuoka 422-8526, Japan \\ ${ }^{3}$ Nutrition Research Department, Food Science Research Laboratories, Meiji Co., Ltd., 540 Naruda, Odawara, Kanagawa 250-0862, Japan
}

(Received 10 January, 2014; Accepted 22 January, 2014; Published online 1 July, 2014)

\begin{abstract}
A dietary combination of sucrose and linoleic acid strongly contributes to the development of metabolic disorders in Zucker fatty rats. However, the underlying mechanisms of the metabolic disorders are poorly understood. We hypothesized that the metabolic disorders were triggered at a stage earlier than the 8 weeks we had previously reported. In this study, we investigated early molecular events induced by the sucrose and linoleic acid diet in Zucker fatty rats by comparison with other combinations of carbohydrate (sucrose or palatinose) and fat (linoleic acid or oleic acid). Skeletal muscle arachidonic acid levels were significantly increased in the sucrose and linoleic acid group compared to the other dietary groups at 4 weeks, while there were no obvious differences in the metabolic phenotype between the groups. Expression of genes related to arachidonic acid synthesis was induced in skeletal muscle but not in liver and adipose tissue in sucrose and linoleic acid group rats. In addition, the sucrose and linoleic acid group exhibited a rapid induction in endoplasmic reticulum stress and abnormal lipid metabolism in skeletal muscle. We concluded that the dietary combination of sucrose and linoleic acid primarily induces metabolic disorders in skeletal muscle through increases in arachidonic acid and endoplasmic reticulum stress, in advance of systemic metabolic disorders.
\end{abstract}

Key Words: arachidonic acid, combination diet, lipotoxicity, insulin sensitivity, ER stress

$\mathrm{D}$ iet is the most important etiological factor involved in the onset of metabolic syndrome and type- 2 diabetes. In particular, excessive calorie intake and chronic consumption of a high fat and/or carbohydrate diet causes obesity and insulin resistance, leading to metabolic syndrome and type- 2 diabetes. At present, the nutritional quality of a diet and the quantities ingested are thought to be important. Consumption of highly glycemic carbohydrates and saturated fatty acids, such as palmitic acid (C16:0), largely contribute to abnormal glucose and lipid metabolism independently of over-nutrition. In addition, since diet per se contains a large number of nutrients, the combined effects of dietary carbohydrate and fat could be added to the individual effects as nutritional interactions. However, to our knowledge, little is known about the relationship between the implications of nutritional interactions and the development of metabolic disorders.

Previously, we demonstrated that a dietary combination of sucrose and linoleic acid (SL) could contribute strongly to the development of metabolic disorders in Zucker fatty rats at 8 weeks compared with other isocaloric diets containing different combinations of carbohydrate and fat, including more ameliorative palatinose (also known as isomaltulose, a sucrose analogue) and oleic acid (C18:1 n-9).(1) We hypothesized that metabolic abnormalities may have already occurred before the 8 weeks. Investigation of this earlier event is necessary to understand the underlying mechanisms of metabolic abnormalities or beneficial effects caused by a combination of dietary carbohydrate and fat. Given the similar obese phenotype in all dietary groups in the previous study, the adverse effects of the SL diet are due to obesityindependent mechanisms. ${ }^{(1)}$ Excessive lipid accumulation in nonadipose tissue, such as the liver, skeletal muscle, and pancreas, is strongly associated with cellular dysfunction and damage as "lipotoxicity", while the particular intracellular lipids present have been paid little attention to date. Matsuzaka et al. ${ }^{(2)}$ reported that metabolic abnormalities, such as insulin resistance and hyperglycemia, could be ameliorated by the modification of hepatic fatty acid composition in Elovl6-deficient mice, even in the presence of persistent obesity and hepatosteatosis. In addition, specific alteration in tissue lipid composition has been shown to be closely involved in cellular dysfunction and metabolic disorders. ${ }^{(3,4)}$

Linoleic acid is an essential $n-6$ polyunsaturated fatty acid (PUFA) with physiological significance due to its conversion to arachidonic acid (C20:4 n-6). A fat-1 transgenic mouse, which expresses the $C$. elegans fat- 1 gene encoding an $n$-3 fatty acid desaturase that converts $n-6$ to $n-3$ fatty acids, can lead to abundant $n-3$ fatty acids with reduced levels of $n-6$ fatty acids in organs and tissues. ${ }^{(5)}$ This model is resistant to a high fat diet and streptozotocin-induced metabolic complications due to the reduction in $n-6$ linoleic and arachidonic acid concentration in their organs. ${ }^{(6,7)}$ These findings strongly suggest that compositional changes in $n$-6 PUFAs in peripheral tissues play a crucial role in the development of metabolic disorders. There is evidence that a dietary high $n-6 / n-3$ PUFA ratio is associated with an increase in the prevalence of diabetes and cardiovascular disease. ${ }^{(8,9)}$ In practice, the fatty acid composition of plasma and peripheral tissues are primarily affected by dietary fatty acids, ${ }^{(10-12)}$ and by carbohydrates to a lesser extent. ${ }^{(13)}$ Sucrose is more readily digestible and ingestible than palatinose and individuals who consume

*To whom correspondence should be addressed.

E-mail: taketani@nutr.med.tokushima-u.ac.jp

tThese authors contributed equally to this work. 
a sucrose-rich diet often present with postprandial hyperglycemia. ${ }^{(14,15)}$ Excessive glucose influx and/or compensatory hyperinsulinemia also affects fatty acid metabolism in peripheral tissues.

In this study, we investigated early molecular events to understand the mechanism by which simultaneous consumption of sucrose and linoleic acid can cooperatively modify tissue fatty acid composition, resulting in progressive metabolic disorders in Zucker fatty rats.

\section{Materials and Methods}

Animal and experimental design. All the animal procedures were performed in accordance with guidelines for the care and use of animals at the University of Tokushima as previously described. ${ }^{(1)}$ Briefly, 11-week old, male Zucker fatty $(f a / f a)$ rats (Charles River, Kanagawa, Japan) were randomly divided into 1 of 4 dietary groups and given an isocaloric diet containing an abundance of (1) palatinose and oleic acid (PO), (2) palatinose and linoleic acid (PL), (3) sucrose and oleic acid (SO), and (4) sucrose and linoleic acid (SL), for 4 weeks under PO group-based pair-feeding. All other components were almost identical between the groups. The detailed composition of experimental diets has been previously described. ${ }^{(1)}$

Oral glucose tolerance test (OGTT) and insulin tolerance test (ITT). The OGTT was performed after 3 and 4 weeks. Rats were fasted for $12 \mathrm{~h}$ before glucose $(2 \mathrm{~g} / \mathrm{kg} \mathrm{BW})$ was orally administrated. ITT was performed at 4 weeks: insulin $(0.75 \mathrm{U} / \mathrm{kg}$ BW, Novolin R, Novo Nordisk, Bagsvaerd, Denmark) was intraperitoneally administrated to the unanesthetized rats. Plasma glucose and insulin levels were determined in blood samples collected from the tail vein at the time point indicated in Fig. 1.

In vivo 2-deoxy-D-glucose uptake (2-DG) assay. In vivo 2-DG uptake in peripheral tissues at 4 weeks was determined using a commercial non-radioisotope 2-DG measurement kit (Cosmo Bio, Tokyo, Japan), according to a previous report. ${ }^{(16)}$ Briefly, rats were injected through a jugular vein catheter with non-radiolabeled 2-DG $(20 \mu \mathrm{l} / \mathrm{kg} \mathrm{BW})$ after $12 \mathrm{~h}$ fasting. Insulin $(0.75 \mathrm{U} / \mathrm{kg} \mathrm{BW})$ was injected into the same catheter $10 \mathrm{~min}$ before the 2-DG injection. After $20 \mathrm{~min}$, the rats were rapidly killed by exsanguination, tissue samples were dissected and quickly frozen with liquid nitrogen and stored at $-80^{\circ} \mathrm{C}$ until analysis.

Tissue fatty acid composition. Whole lipid was extracted from snap-frozen tissue samples with water/chloroform/methanol $(0.7: 1: 1, \mathrm{vol} / \mathrm{vol} / \mathrm{vol})$ containing butylated hydroxytoluene as an antioxidant, according to the method of Bligh and Dyer. ${ }^{(17)}$ The extracted lipids were trans-methylated with $\mathrm{HCl}$-methanol at $100^{\circ} \mathrm{C}$ for $2 \mathrm{~h}$. The fatty acid methyl esters were separated using gas-liquid chromatography (GC-18A, Shimadzu, Kyoto, Japan) with a capillary column (SP2330, Supelco, Bellefonte, PA). Individual fatty acids were identified by comparing the retention time of each peak with those of the internal standards.

RNA preparation and quantitative RT-PCR. Total RNA was extracted and then RT-PCR reactions were performed as previously described.(1) The sequence of gene-specific primers are listed in our previous report. ${ }^{(1)}$ Additional primers used in this study are listed in Table 1.

Immunoblotting. Total protein was extracted from snapfrozen tissue samples using lysis buffers and inhibitors for both protease and phosphatase (Nacalai Tesque, Kyoto, Japan). Samples were denatured by heating at $95^{\circ} \mathrm{C}$ for $5 \mathrm{~min}$ in a sample buffer in the presence of 5\% 2-mercaptoethanol. Proteins were separated by SDS-PAGE and transferred onto PVDF membrane (Immobilon$\mathrm{P}$, Millipore, Bedford, MA) by electrophoresis. Protein-bound membranes were treated with target-specific protein antibodies to p-Akt (Ser473), total-Akt, p-JNK (Thr183/185), total JNK p-AMPK $\alpha$ (Thr172), total AMPK $\alpha, \beta$-actin (Cell Signaling Technology, Beverly, MA), and SREBP-1c (Santa Cruz Biotech- nology, Santa Cruz, CA) and visualized using a luminescent image analyzer (LAS-3000UVmini, Fujifilm, Tokyo, Japan). The data were also quantified using Malti Gauge software ver. 3.0 (Fujifilm).

Statistical analysis. Results were expressed as means \pm SEM. The significance of differences between the groups was determined by ANOVA or the nonparametric Kruskal-Wallis test, followed by a post-hoc test. The significant effects of differences in dietary carbohydrate and fat were identified by 2-way ANOVA followed by the Tukey-Kramer post-hoc test. These statistical calculations were performed using Excel Toukei 2006 (SSRI, Tokyo, Japan) and Graph Pad Prism ver. 5 (Graph Pad Software, San Diego, CA). Differences with a $p$ value $<0.05$ were considered statistically significant.

\section{Results}

Glucose tolerance and whole body insulin sensitivity.

After 4-weeks of feeding, the metabolic phenotypes, including body weight, visceral fat content, plasma glucose, triacylglyceride (TG), adipokines and hepatic TG content were similar between the groups (Table 2). However, OGTT revealed that plasma insulin levels were consistently higher in the high-linoleic acid groups (PL and SL) compared with the high-oleic acid groups (PO and SO) at 3 weeks: plasma glucose levels showed little difference between the groups, except for a small increase in blood glucose levels at $120 \mathrm{~min}$ in the SL group (Fig. 1A and B). Plasma glucose levels in the SL group remained lower relative to the reduced insulin secretion during OGTT at 4 weeks (Fig. 1C). It is noteworthy that plasma insulin levels were dramatically decreased in the SL group at 4 weeks (Fig. 1D). At the same time, ITT revealed that the SL group was modestly sensitive to exogenous insulin compared with the other groups (Fig. 1E). Overall, the investigation at 4 weeks was considered to be sufficient to demonstrate early molecular events induced by the SL diet in Zucker fatty rats.

In vivo glucose uptake in peripheral tissues. To elucidate the effects of each diet on peripheral insulin sensitivity, in vivo 2DG uptake in the peripheral tissues was determined under insulin stimulation. The results demonstrated that 2-DG uptake tended to be enhanced both in the skeletal muscle (gastrocnemius and soleus) and epididymal white adipose tissue (eWAT) in the SL group compared to other groups (Fig. 2A-C). In addition, hepatic 2-DG uptake was significantly enhanced in the SL group compared to the PO and SO groups (Fig. 2D). Akt phosphorylation at Ser473 was highest in all tissues isolated from the SL group in the presence of insulin administration (Fig. 3A-D). Taken together, the phenotype of the SL group was characterized by both decreased insulin secretion and increased peripheral insulin sensitivity at 4 weeks.

Fatty acid composition in peripheral tissues. Fatty acid composition of the liver, soleus muscle, and eWAT was determined by gas-liquid chromatography. As expected, the tissue fatty acid profile was directly affected by fatty acid composition in each diet: oleic acid levels were increased in the PO and SO groups, while linoleic acid levels were increased in the PL and SL groups (Fig. 4A-C). In addition to the dietary fatty acid composition, the type of dietary carbohydrate was, at least in part, another determinant of the tissue fatty acid profile (Fig. 4A-C). We also found tissue-specific differences in arachidonic acid levels between the liver and soleus muscle, however, levels in eWAT were modified by dietary combinations of carbohydrate and fat (Fig. 4A-C). Arachidonic acid levels were significantly increased in both tissues in the SL group compared to other groups at 4 weeks (Fig. 4A and B).

Tissue-specific change in glucose and lipid metabolism.

To evaluate the influence of the alteration in fatty acid profile on cellular glucose and lipid metabolism, we examined gene expression in relation to glucose and lipid metabolism in peri- 

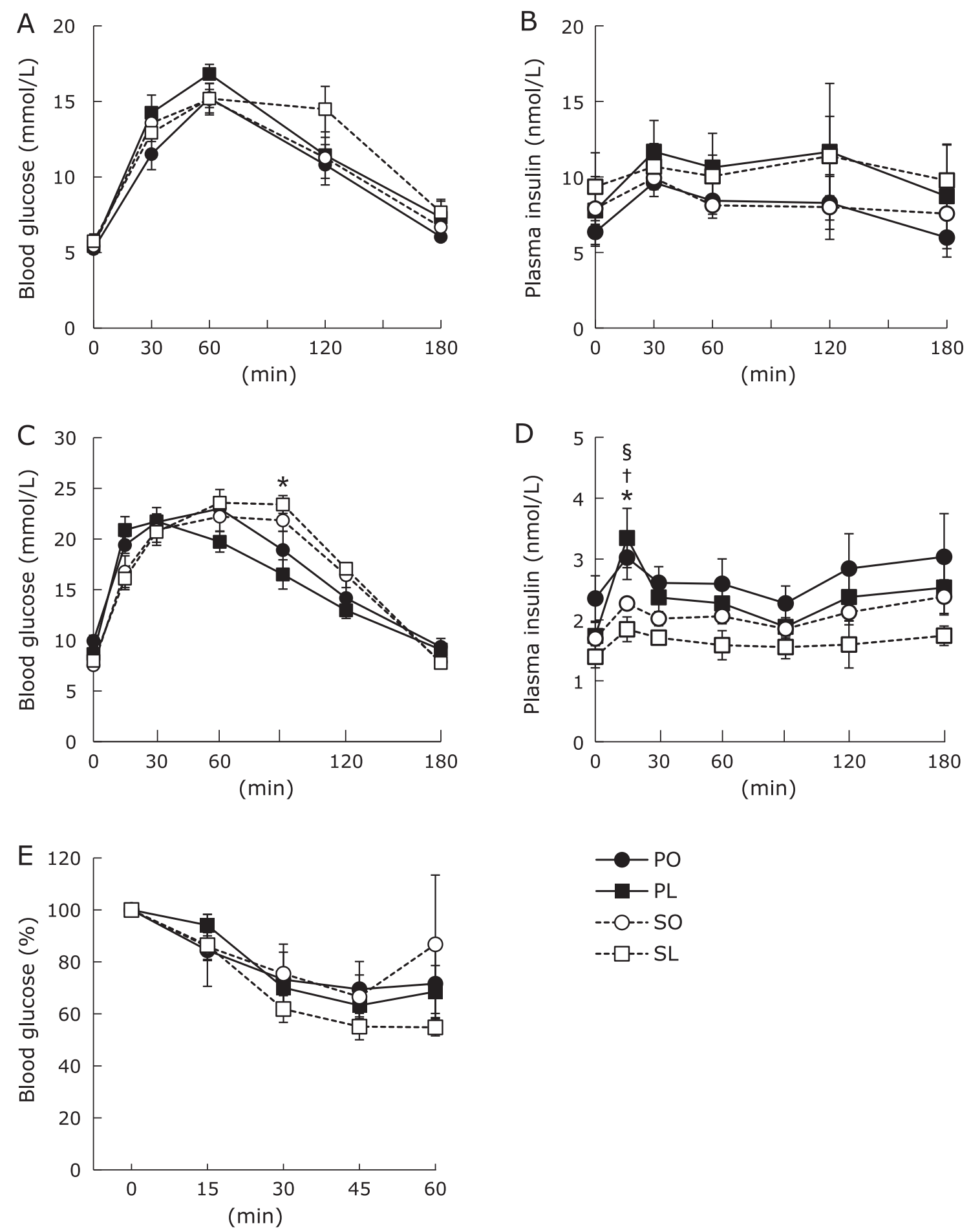

Fig. 1. Glucose tolerance and whole body insulin sensitivity. OGTT was performed at 3 weeks (A and B) and 4 weeks (C and D). Plasma glucose and insulin levels were measured in a blood sample collected at each indicated time after glucose administration ( $2 \mathrm{~g} / \mathrm{kg} \mathrm{BW}$ ). (E) ITT was performed at 4 weeks. Plasma glucose levels at each indicated time were represented as the percent of the value at time 0 . Results were means \pm SEM $(n=5-6)$. ${ }^{*} p<0.05$ vs PL group for SL group, ${ }^{\dagger} p<0.05$ vs PO group for PL group, and ${ }^{\S} p<0.05$ vs PL group for SO group.

pheral tissues. In the soleus muscle, lipogenic gene expression was remarkably increased in the SL group (Fig. 5A). More importantly, the mRNA levels of delta-5 desaturase (D5D) and fatty acid elongase 5 (Elov15), which are enzymes converting linoleic acid to arachidonic acid, and fatty acid translocase/cluster of differentiation 36 (FAT/CD36), respectively, were also significantly increased in the SL group but not the PL group (Fig. 5A). However, we did not find similar induction levels of genes involved in glucose and lipid metabolism in the liver and eWAT (Fig. 5C). Induction of key transcription factors in lipid synthesis, sterolregulatory element binding protein 1c (SREBP1c) and carbohydrate responsive element binding protein (ChREBP), may be partly responsible for the induction of lipogenesis genes in the soleus muscle by the SL diet (Fig. 5A and D). The SL group also 
Table 1. Sequence of oligonucleotide primers for quantitative RT-PCR analysis

\begin{tabular}{|c|c|c|c|c|}
\hline \multirow{2}{*}{ Gene name } & \multirow{2}{*}{ Size (bp) } & \multirow{2}{*}{ Accession No. } & \multicolumn{2}{|c|}{ Primer sequence } \\
\hline & & & $\begin{array}{l}\text { Forward } \\
\end{array}$ & Reverse \\
\hline ChREBP & 113 & $A B 074517$ & 5'-CAGCTTCTCGACTTGGACTG-3' & 5'-TTGCCAACATAAGCGTCTTC-3' \\
\hline Elovl5 & 150 & NM_134382 & 5'-TTCTTCTGTCAGGGAACACG-3' & 5'-GTGCAGGACTGTGATCTGGT-3' \\
\hline D5D & 178 & NM_053445 & 5'-AAGCACATGCCATACAACCA-3' & 5'-CAGCGGCATGTAAGTGATGA-3' \\
\hline D6D & 144 & NM_031344 & 5'-ACATCATCGTCATGGAAAGC-3' & 5'-CAGAAAGGTGGCCATAATCA-3' \\
\hline ATGL & 148 & NM_001108509 & 5'-GAGATGTGCAAACAGGGCTA-3' & 5'-CAGTCCTCTCCTCAGTCACG-3' \\
\hline DGAT & 150 & NM_053437 & 5'-GACAGCGGTTTCAGCAATTA-3' & 5'-GGGGTCCTTCAGAAACAGAG-3' \\
\hline FATP & 100 & U89529 & 5'-AGGTGACGTGCTAGTGATGG-3' & 5'-CTCCGTGGTGGATACGTTCT-3' \\
\hline GPAT & 116 & NM_017274 & 5'-GTTATCAGAATGCTGCGGAA-3' & 5'-GAGAGGGGAGCAGATACAGG-3' \\
\hline FAT/CD36 & 145 & NM_031561 & 5'-ATGCCGGTTGGAGACCTACT-3' & 5'-CCTCTGGGTTTTGCACGTCA-3' \\
\hline FABP & 174 & NM_024162 & 5'-CATGAAGTCACTCGGTGTGG-3' & 5'-TCATCTGCTGTGACCTCGTC-3' \\
\hline Elovl6 & 99 & NM_134383 & 5'-AGCAAAGCACCCGAACTAGG-3' & 5'-GTACAGGAGCACAGTGATGTGG-3' \\
\hline 5-LOX & 198 & NM_012822 & 5'-CCCATCTGCCTGCTGTATAA-3' & 5'-CAGATGTGTGCGGAGAAGAT-3' \\
\hline 12-LOX & 92 & L06040 & 5'-TCTGGCAGATCATGAATCGG-3' & 5'-GCTCTGCAGTTCATAGTCGT-3' \\
\hline PPAR $\gamma$ & 147 & AF156665 & 5'-TCAAACCCTTTACCACGGTT-3' & 5'-CAGGCTCTACTTTGATCGCA-3' \\
\hline TNF- $\alpha$ & 143 & NM_012675 & 5'-ATGGATCTCAAGACAACCA-3' & 5'-TCCTGGTATGAAATGGCAAA-3' \\
\hline MCP-1 & 93 & M57441 & 5'-CCCTAAGGACTTCAGCACCTTTG-3' & 5'-AAGTGCTTGAGGTGGTTGTGG-3' \\
\hline Acrp30 & 140 & NM_144744 & 5'-GGAAACTTGTGCAAGGTTGGA-3' & 5'-GGTCACCCTTAGGACCAAGA-3' \\
\hline Bip & 279 & NM_013083 & 5'-CCTGTTGCTGGACTCTGTGA-3' & 5'-AGGAGTGAAGGCCACATACG-3' \\
\hline Chop & 231 & NM_001109986 & 5'-TTGGGGGCACCTATATCTCA-3' & 5'-CGCACTGACCACTCTGTTTC-3' \\
\hline Xbp1 & 229 & NM_001004210 & 5'-GAGCAGCAAGTGGTGGATTT-3' & 5'-GAGGCGCACGTAGTCTGAGT-3' \\
\hline Atf3 & 225 & NM_012912 & 5'-CCAGGTCTCTGCCTCAGAAG-3' & 5'-GCCACCTCAGACTTGGTGA-3' \\
\hline Atf6 & 249 & NM_001107196 & 5'-AGCAGAGCCACTGAAGGAAG-3' & 5'-GGGGTCCTTCAGAAACAGAG-3' \\
\hline LPCAT3 & 188 & NM_001012189 & 5'-GATGGCCTACCTTCTTGCTG-3' & 5'-ATGAGGGGACACCCAGTATG-3' \\
\hline LPIAT1 & 177 & NM_001134978 & 5'-ATTGCAGCTTCCCTGGAGTA-3' & 5'-GGCACTCCTCAAAACGTAGG-3' \\
\hline SERCA1 & 170 & NM_058213 & 5'-AAAGGAAGGAGCCCAGGTTA-3' & 5'-GCTCACCCCAAAATAGGACA-3' \\
\hline SERCA2a & 228 & NM_001110823 & 5'-TATTGGCTGGTGAAGGAGGT-3' & 5'-GACAATGTCTGCTGGCTCAA-3' \\
\hline SERCA2b & 176 & NM_001110139 & 5'-CCGCTTCCTAAACATTGCAG-3' & 5'-CAACAGCTAAAACCCCACTTG-3' \\
\hline
\end{tabular}

Table 2. Body weight, plasma concentrations, and hepatic lipid content of Zucker fatty rats fed each diet for 4 weeks

\begin{tabular}{|c|c|c|c|c|}
\hline & $\mathrm{PO}$ & $\mathrm{PL}$ & SO & SL \\
\hline Body weight (g) & $607.21 \pm 11.21$ & $600.76 \pm 17.36$ & $624.62 \pm 13.25$ & $621.15 \pm 11.40$ \\
\hline Visceral fat (g/kg BW) & $137.34 \pm 4.54$ & $135.15 \pm 6.63$ & $142.92 \pm 3.72$ & $142.62 \pm 3.61$ \\
\hline Mesenteric fat & $37.13 \pm 1.42$ & $37.35 \pm 2.15$ & $41.10 \pm 1.48$ & $39.49 \pm 1.85$ \\
\hline Epididymal fat & $38.41 \pm 1.89$ & $36.93 \pm 2.47$ & $42.38 \pm 2.36$ & $40.74 \pm 1.60$ \\
\hline Retroperitoneal fat & $61.80 \pm 5.38$ & $60.87 \pm 3.33$ & $59.44 \pm 1.34$ & $62.44 \pm 1.60$ \\
\hline \multicolumn{5}{|l|}{ Plasma concentrations } \\
\hline Glucose (mmol/L) & $7.68 \pm 0.15$ & $6.55 \pm 0.46$ & $7.04 \pm 0.31$ & $8.07 \pm 1.07$ \\
\hline Insulin (nmol/L) & $1.91 \pm 0.21$ & $1.62 \pm 0.25$ & $1.31 \pm 0.09$ & $2.13 \pm 0.43$ \\
\hline $\mathrm{TG}(\mathrm{mg} / \mathrm{dL})$ & $147 \pm 14$ & $166 \pm 23$ & $215 \pm 37$ & $150 \pm 18$ \\
\hline $\mathrm{T}-\mathrm{Chol}(\mathrm{mg} / \mathrm{dL})$ & $162 \pm 14$ & $159 \pm 15$ & $226 \pm 24 *$ & $171 \pm 17$ \\
\hline NEFA $(m E q / L)$ & $0.68 \pm 0.05$ & $0.76 \pm 0.05$ & $0.74 \pm 0.06$ & $0.74 \pm 0.05$ \\
\hline Adiponectin $(\mu \mathrm{g} / \mathrm{mL})$ & $10.18 \pm 3.83$ & $9.25 \pm 3.19$ & $13.56 \pm 2.18$ & $13.57 \pm 3.81$ \\
\hline Leptin $(p g / m L)$ & $127.4 \pm 23.5$ & $93.6 \pm 21.1$ & $131.0 \pm 14.4$ & $129.0 \pm 10.5$ \\
\hline Hepatic TG (mg/g tissue) & $107 \pm 5.0$ & $128 \pm 18$ & $121 \pm 15$ & $128 \pm 18$ \\
\hline
\end{tabular}

Values are mean \pm SEM $(n=6) .{ }^{*} p<0.05$ vs PO, PL, SL groups for SO group.

showed the lowest phosphorylation of 5'AMP-activated protein kinase (AMPK) in the soleus muscle (Fig. 5E). In contrast, hepatic mRNA expression of D5D and delta-6 desaturase (D6D) was decreased in the PL and SL groups (Fig. 5B), indicating a physiological response to maintain a balance of hepatic fatty acid composition.

Endoplasmic reticulum (ER) stress response in skeletal muscle. An imbalance between de novo lipogenesis and fatty acid oxidation causes cellular dysfunction in skeletal muscle through lipotoxicity-induced cellular responses, such as ER stress. ${ }^{(18,19)}$ As expected by the enhanced lipogenesis, mRNA expression of ER stress-inducible genes, such as binding immuno- globulin protein (Bip), C/EBP homologous protein (Chop), and $\mathrm{X}$-box binding protein 1 (Xbpl) was remarkably increased in the $\mathrm{SL}$ group compared to the other groups (Fig. 6A). c-Jun $\mathrm{NH}_{2}-$ terminal kinase (JNK) plays an important role in the development of insulin resistance in response to inflammation and ER stress. ${ }^{(20,21)}$ We also found that the SL diet could promote JNK activation in the soleus muscle in the presence of the induction of ER stress after 4 weeks of feeding (Fig. 6B).

Phospholipid remodelling in skeletal muscle ER. Finally, we focused on whether a compositional change in phospholipids is involved in the induction of ER stress in the soleus muscle. A member of the membrane-bound $O$-acyltransferase (MBOAT) 
A Gastrocnemius

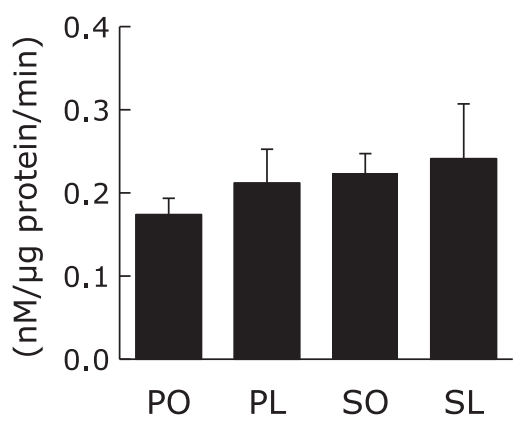

C EWAT

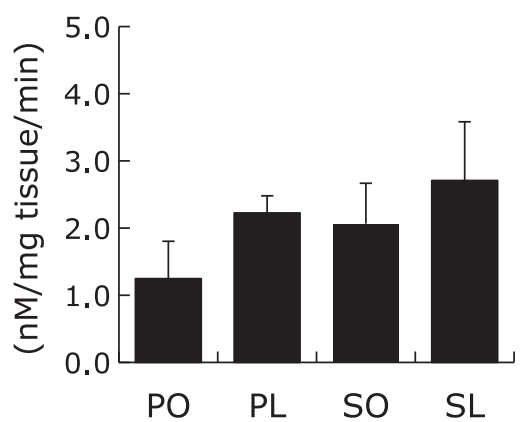

B Soleus

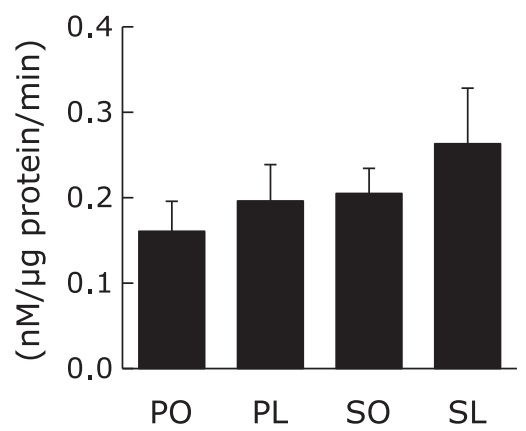

D Liver

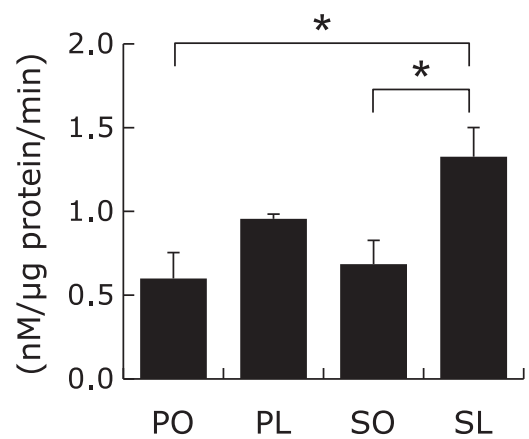

Fig. 2. In vivo glucose uptake in peripheral tissues at 4 weeks. Insulin-stimulated 2-DG uptake in gastrocnemius (A), soleus (B), epididymal adipose tissue (eWAT) (C), and liver (D) were determined by non-radioisotopic assay. Results were means \pm SEM $(n=4-6)$. ${ }^{\star} p<0.05$.

family encoding lipid remodeling enzymes, lysophosphatidylcholine acyltransferase 3 (LPCAT3) and lysophosphatidylinositol acyltransferase 1 (LPIAT1, also known as MBOAT7) have been identified as key enzymes incorporating PUFA and arachidonic acid into phospholipid species. ${ }^{(22)}$ The SL group showed a trend towards higher LPCAT3 and LPIAT1 mRNA expression as well as liver $\mathrm{X}$ receptor (LXR) $\alpha$ compared with the other groups (Fig. 6A). Compositional changes in membrane phospholipids can affect the membrane fluidity and the activity of proteins which are located in the phospholipid membrane. Sarco/endoplasmic reticulum $\mathrm{Ca}^{2+}$-ATPase (SERCA) is a $\mathrm{Ca}^{2+}$ pump localized in the sarcoplasmic reticulum (SR)/ER membrane which transports $\mathrm{Ca}^{2+}$ from the cytosol to the SR/ER lumen against a large gradient of concentration. In this study, mRNA expression levels of SERCA1 tended to increase in the SL group to levels similar to LPCAT3 and LPIAT1, however, mRNA expressions of SERCA2a and SERCA2b did not follow this trend (Fig. 6A).

\section{Discussion}

We have previously reported that 8-weeks feeding of the SL diet could strongly contribute to the development of metabolic complications in Zucker fatty rats. ${ }^{(1)}$ In the present study, we focused on studying the early molecular events induced by the adverse interactions between carbohydrate and fat. Here, we demonstrate that the adverse consequences of the SL diet may be preceded by changes in the fatty acid profile of peripheral tissues. In particular, arachidonic acid levels were significantly increased in the liver and soleus muscle in the SL group at 4 weeks. However, a significant increase in the mRNA expression of Elovl5, D5D, and FAT/CD36 was observed only in the soleus muscle at 4 weeks. These findings indicate that the activation of arachidonic acid biosynthesis and cellular uptake may be involved in the abundance of arachidonic acid in the skeletal muscle. The elevated hepatic arachidonic acid may simply reflect plasma arachidonic acid levels (data not shown), because D5D and Elovl5 did not increase in the liver at 4 weeks. In addition, mRNA expression of other lipogenic genes, such as ACC and SCD-1, was also significantly increased in the skeletal muscle in the SL group. Therefore, skeletal muscle lipid metabolism is considered to be more susceptible to the interactions between dietary carbohydrate and fat in Zucker fatty rats. There is evidence that membrane fatty acid composition in skeletal muscle may be most responsive to the balance of dietary $n-6$ and $n-3$ PUFA. ${ }^{(23)}$ Generally, oral administration of linoleic acid can endogenously increase arachidonic acid levels in membrane phospholipids. ${ }^{(10-12)}$ Arachidonic acid biosynthesis is often augmented under hyperglycemic conditions, which in turn links to cellular dysfunction. ${ }^{(24,25)}$ The SL diet-induced gene expression in skeletal muscle is consistent with the previous study identifying early inducible genes in skeletal muscle in response to physiological superimposing hyperglycemia and hyperinsulinemia. ${ }^{(26)}$ A sucrose-rich diet has a higher glycemic index than a palatinose-rich diet, ${ }^{(15)}$ and linoleic acid and arachidonic acid per se have been reported to have an insulinotropic effect on pancreatic beta-cells. ${ }^{(27-30)}$ Our results suggest that simultaneous ingestion of sucrose and linoleic acid could potentially synchronize both hyperglycemia and hyperinsulinemia. These physiological responses in turn have a large impact on skeletal muscle glucose and lipid metabolism, leading to the activation of lipogenesis and an increase in arachidonic acid levels.

Furthermore, the SL group exhibited a rapid induction in ER stress in the skeletal muscle. An imbalance of phospholipid species and constituent fatty acids can be implicated in ER stress and insulin resistance. ${ }^{(18,19)}$ Funai $\mathrm{K}$ et al. ${ }^{(31)}$ reported that muscle lipogenesis regulates cytosolic $\mathrm{Ca}^{2+}$ levels by phospholipid remodeling in the SR membrane to regulate insulin sensitivity 


\section{A Gastrocnemius}
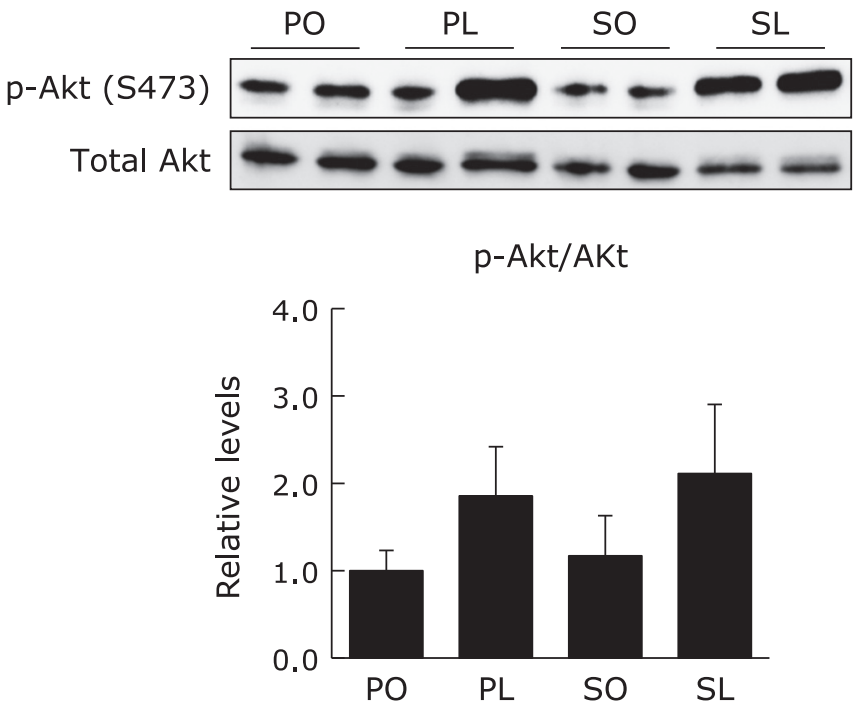

C eWAT
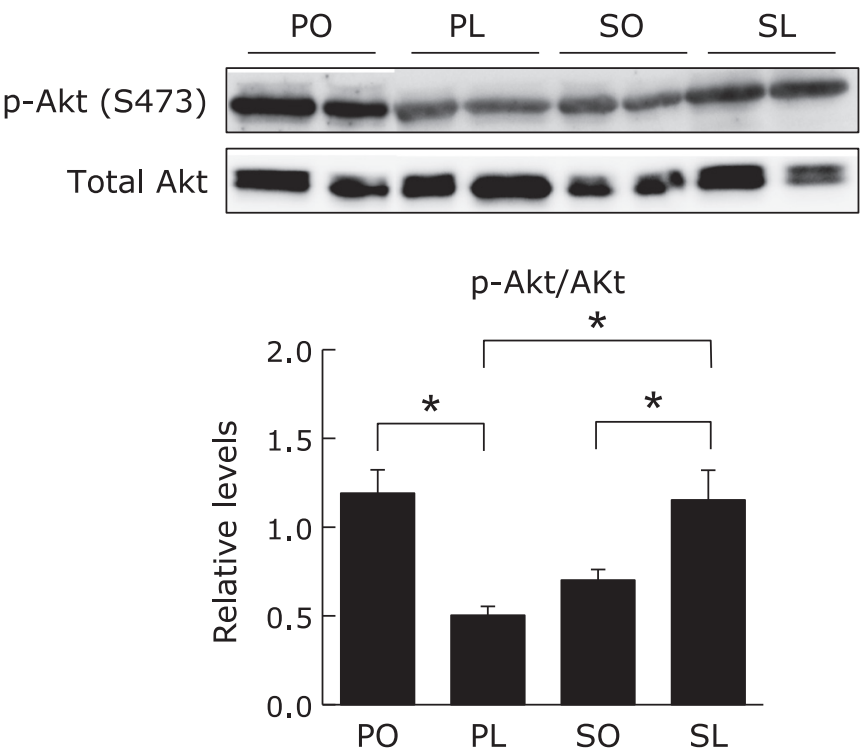

B Soleus

p-Akt (S473)

Total Akt
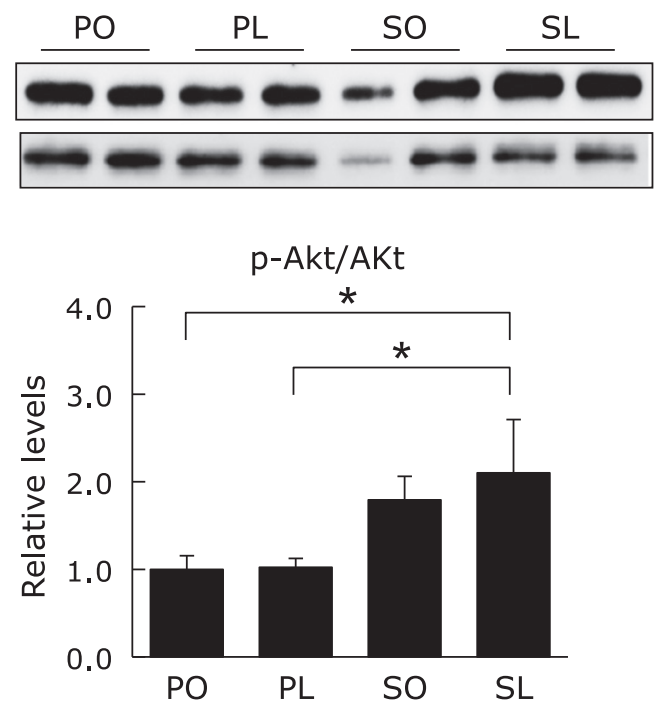

D Liver

p-Akt (S473)

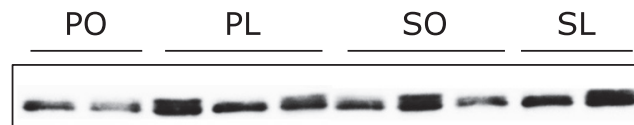

Total Akt
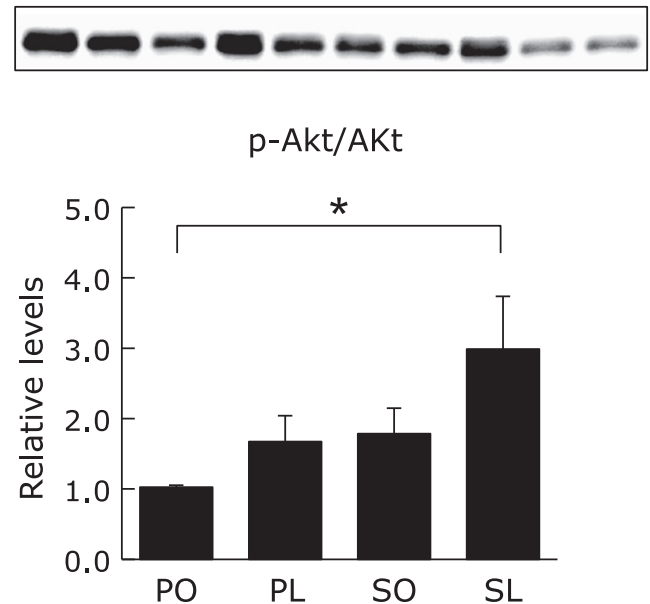

Fig. 3. Akt phosphorylation in peripheral tissues at 4 weeks. Protein samples extracted from gastrocnemius (A), soleus (B), eWAT (C), and liver (D) under insulin stimulation were subjected to immunoblotting for p-Akt (Ser473) and Akt, as indicated. Intensity of the signals was quantified using software (Malti Gauge ver. 3.0). Results were means \pm SEM $(n=5-6)$ and calculated as relative expression levels to the PO group. ${ }^{*} p<0.05$.

through the calcium/calmodulin-dependent protein kinase kinase (CaMKK)-AMPK pathway. SERCA plays a key role in the inactivation of the cytosolic CaMMK-AMPK pathway by transporting $\mathrm{Ca}^{2+}$ from the cytoplasm into the SR lumen. ${ }^{(31)} \mathrm{We}$ found that mRNA expression of SERCA1 was remarkably increased in the SL group compared to the other groups. Correspondingly, AMPK activity was lower in the SL group compared with the other groups. Thus, attenuation of the CaMKK-AMPK pathway, at least in part due to the decrease in cytosolic $\mathrm{Ca}^{2+}$ levels, may be responsible for SL diet-induced abnormal metabolic homeostasis in the skeletal muscle. Given the increased mRNA expression of LPCAT3 and LPIAT1, as well as LXR $\alpha$, in the SL group, preferential incorporation of arachidonic acid into phospholipids of the SR membrane may be an important modification involved in the induction of ER stress and increased SERCA levels. Recently, LXRs have been reported to play an important role in regulating ER stress through LPCAT3-mediated dynamic modulation of membrane phospholipid composition. ${ }^{(32)}$ Moreover, the activation of JNK protein kinase was more prominent in the skeletal muscle of the SL group. In contrast, the PO diet would be a more beneficial combination in the prevention of skeletal muscle metabolic abnormalities at 4 weeks. Collectively, we concluded that these metabolic abnormalities can be followed by insulin resistance in the skeletal muscle of the SL group rats.

Unexpectedly, ITT demonstrated that enhanced glucose uptake was observed in the skeletal muscle, eWAT and liver at 4 weeks in the SL group. This result was supported by the enhancement of Akt phosphorylation at Ser473, which is a downstream signal 


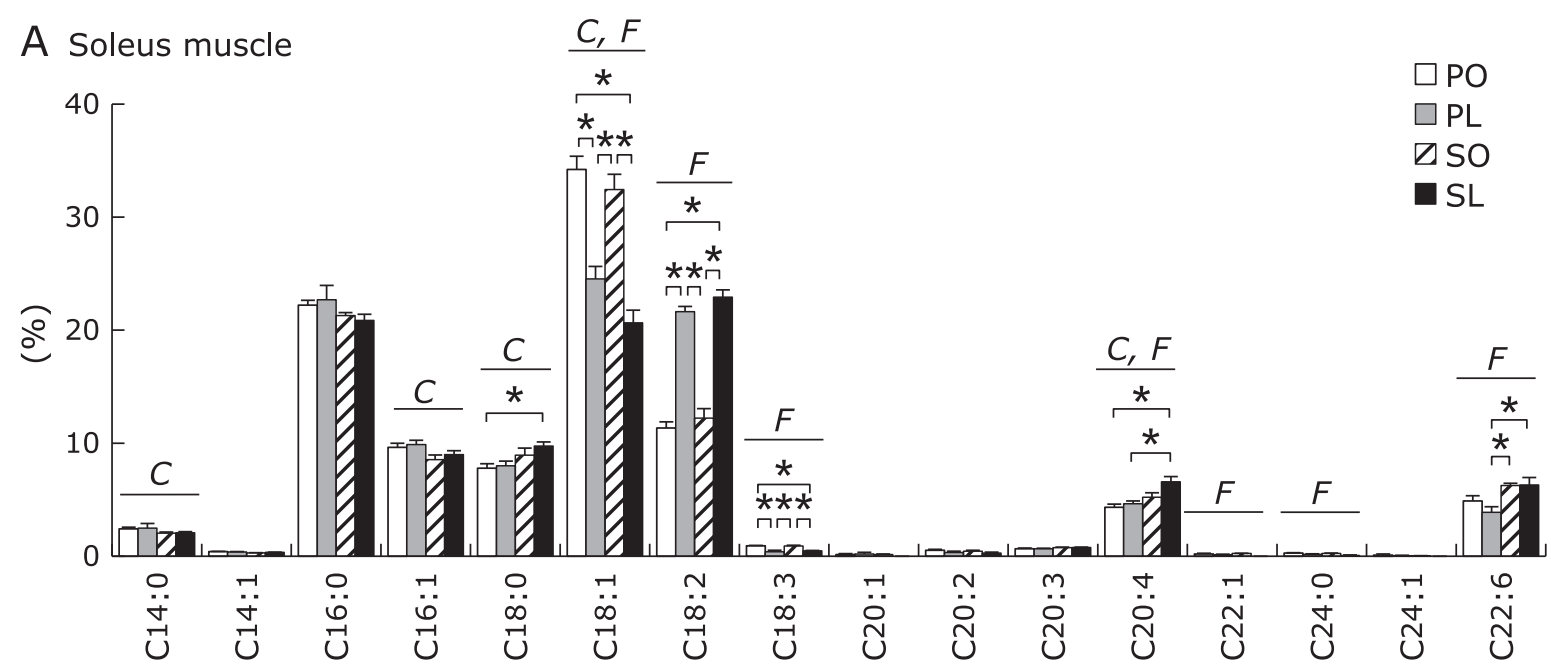

B Liver

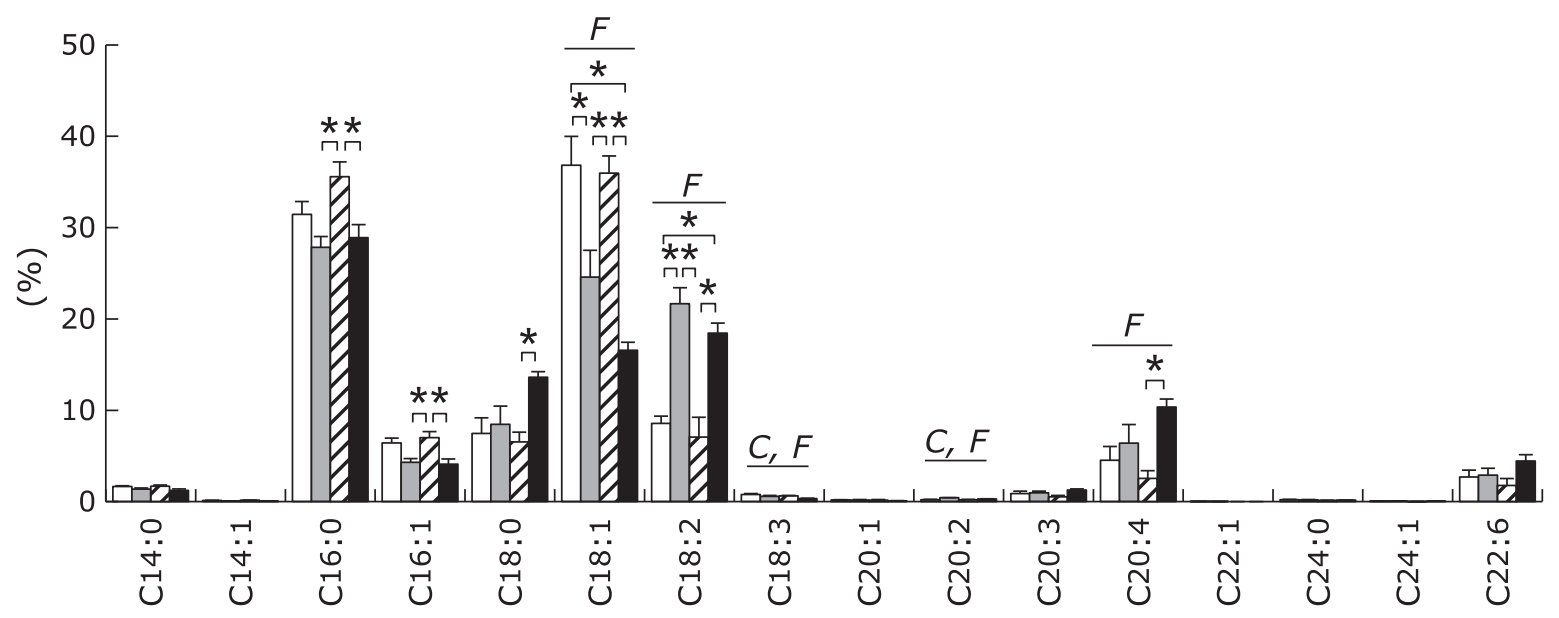

C eWAT

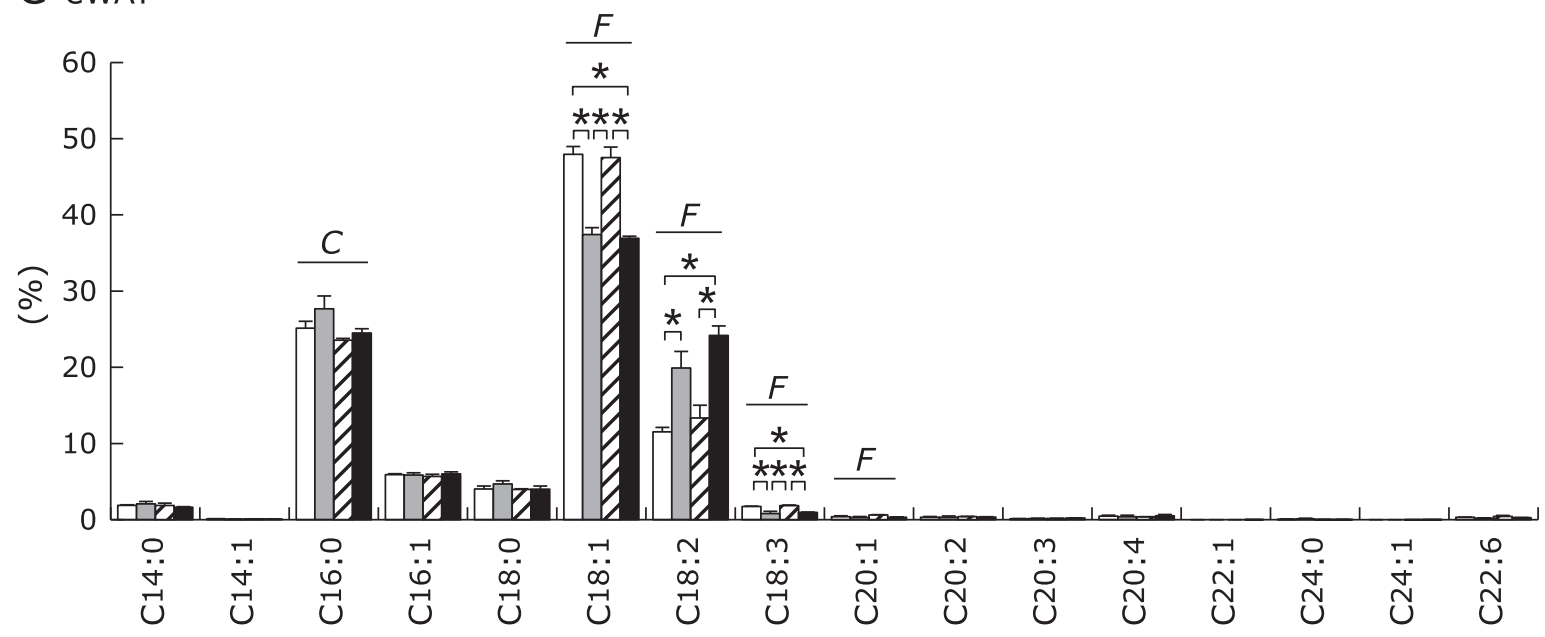

Fig. 4. Fatty acid composition in peripheral tissues at 4 weeks. Fatty acid composition of whole lipid extracted from soleus muscle (A), liver (B), and eWAT (C) were determined by gas-liquid chromatography. Results were means \pm SEM $(n=5-6)$ and represented as a percentage of total fatty acid content. ${ }^{*} p<0.05$. Significant dietary effects of carbohydrate and fat $(p<0.05)$ by 2 -way ANOVA were described as $C$ (palatinose vs sucrose) and $F$ (oleic acid vs linoleic acid). 


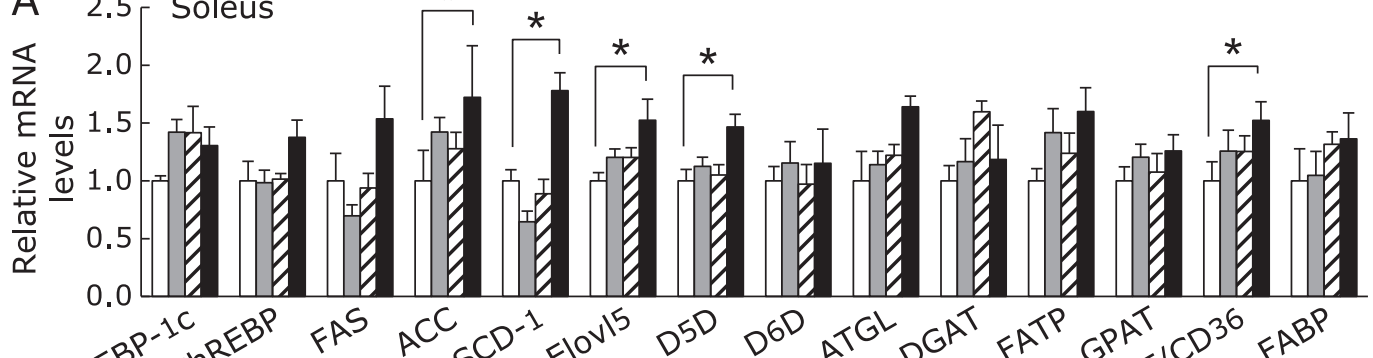

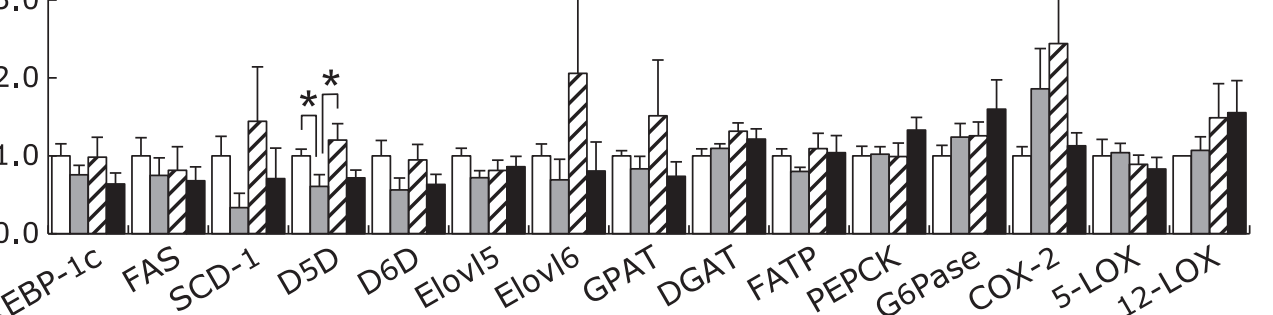

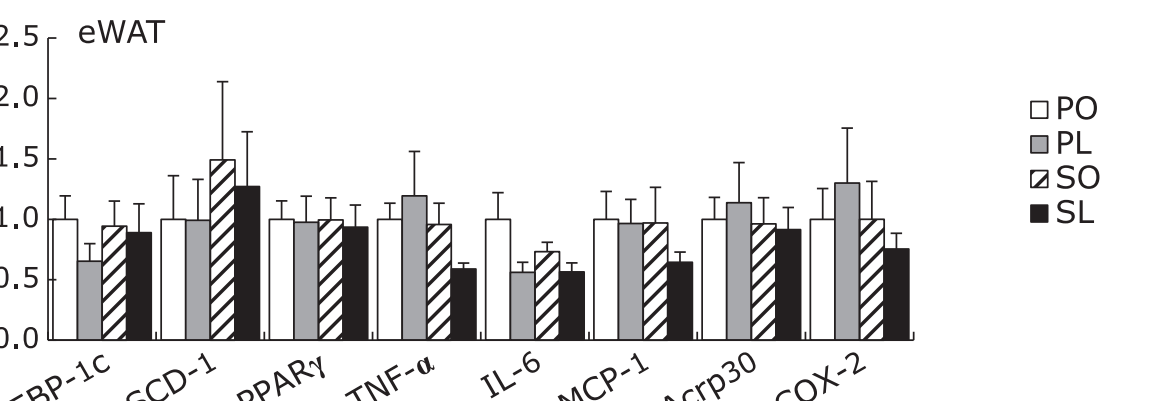

等,

IIII III. 

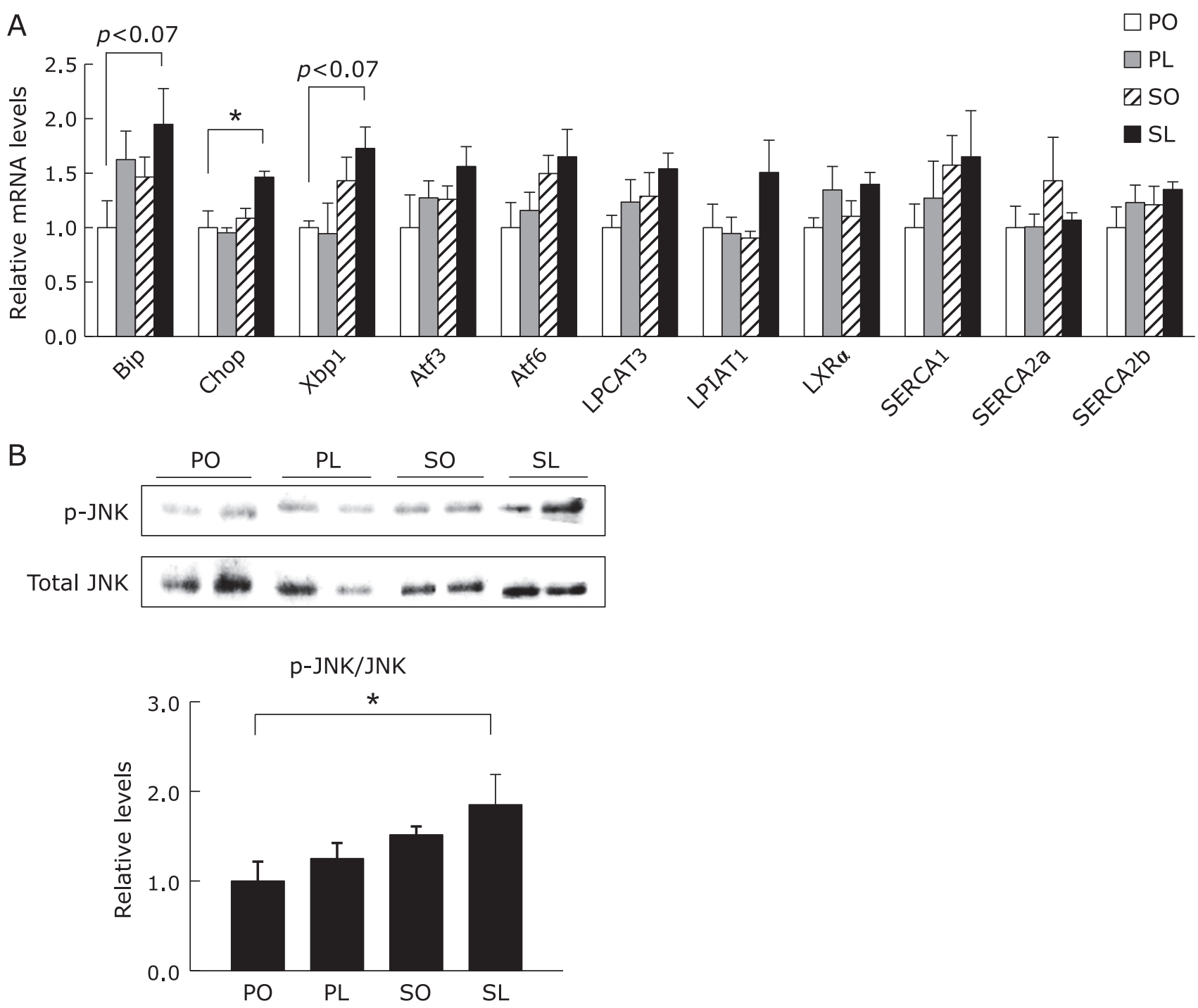

Fig. 6. ER stress response in soleus muscle at 4 weeks. (A) mRNA expression of genes related to ER stress and phospholipid remodeling in soleus muscle was determined by quantitative RT-PCR analysis. (B) Protein samples extracted from soleus muscle were subjected to immunoblotting for $p$ JNK (Thr183/185) and total JNK, as indicated. Results were means \pm SEM $(n=5-6)$ and calculated as relative expression levels to the PO group. ${ }^{*} p<0.05$.

for the insulin receptor. Therefore, skeletal muscle insulin sensitivity was enhanced in the SL group under exogenous insulin stimulation, even in the presence of abnormal lipid metabolism and ER stress. These results may provide a more satisfactory explanation for the conflicting observation during OGTT at 4 weeks that the SL group did not exhibit overt hyperglycemia relative to the decline in plasma insulin levels. It has been reported that arachidonic acid can play another role in stimulating glucose uptake by increasing glucose transporter levels in the plasma membrane and glucose transport activity in adipocytes. ${ }^{(33)}$ It has also been reported that cellular arachidonic acid potentially interacts with phosphoinositide 3-kinase (PI3K)/Akt or phosphatase and tensin homolog (PTEN) and thereby activates its downstream signaling in endothelial and cancer cells. ${ }^{(34-36)}$ These findings suggest that a characteristic distribution of arachidonic acid in peripheral tissues may be physiologically important to maintain systemic glucose homeostasis and normal cellular function. Nevertheless, 8-weeks feeding of the SL diet eventually lead to severe metabolic disorders in Zucker fatty rats, probably due to coincidental impaired insulin secretion. ${ }^{(1,37)}$ Indeed, exogenous insulin administration was required for the high activity of glucose uptake and insulin signaling shown in the SL group. It is noteworthy that elevated arachidonic acid in the skeletal muscle and liver from the SL group was diminished after 8 weeks (data not shown).

Finally, we should mention the responsibility of the absence of leptin signaling in Zucker fatty rats due to a mutation in the leptin receptor gene (Lepr), since peripheral leptin signaling plays an important role in the prevention of lipotoxicity-induced metabolic dysfunction in skeletal muscle and pancreas. ${ }^{(38-41)}$ Our results demonstrate that skeletal muscle and pancreatic islets ${ }^{(37)}$ were an initial target tissue response to the adverse dietary interactions of the SL diet in Zucker fatty rats. Further research using other animal models is needed to determine the effect of deficient peripheral leptin signaling on the molecular mechanisms reported.

In conclusion, we demonstrate that the adverse consequences of a dietary combination of sucrose and linoleic acid in Zucker fatty rats can be initiated by abnormal lipid metabolism in the skeletal muscle. Although the underlying mechanism is still under investigation, an abnormal accumulation of cellular arachidonic acid 
may be involved in the molecular basis of metabolic abnormalities in the skeletal muscle. Our findings suggest that a selective combination between dietary carbohydrates and fats can induce compositional and distributional changes in intracellular fatty acids in specific tissues, resulting in the development of metabolic disorders.

\section{Acknowledgments}

We thank Prof. Junji Terao (Department of Food Science, The University of Tokushima) for providing access to GC-MS, and

\section{References}

1 Sato K, Arai H, Mizuno A, et al. Dietary palatinose and oleic acid ameliorate disorders of glucose and lipid metabolism in Zucker fatty rats. J Nutr 2007; 137: 1908-1915.

2 Matsuzaka T, Shimano H, Yahagi N, et al. Crucial role of a long-chain fatty acid elongase, Elovl6, in obesity-induced insulin resistance. Nat Med 2007; 13: 1193-1202.

3 Borkman M, Storlien LH, Pan DA, Jenkins AB, Chisholm DJ, Campbell LV. The relation between insulin sensitivity and the fatty-acid composition of skeletal-muscle phospholipids. N Engl J Med 1993; 328: 238-244.

4 Pan DA, Lillioja S, Milner MR, et al. Skeletal muscle membrane lipid composition is related to adiposity and insulin action. J Clin Invest 1995; 96: 2802-2808.

5 Kang JX, Wang J, Wu L, Kang ZB. Transgenic mice: fat- 1 mice convert $n-6$ to $n-3$ fatty acids. Nature 2004; 427: 504.

6 White PJ, Arita M, Taguchi R, Kang JX, Marette A. Transgenic restoration of long-chain $n$-3 fatty acids in insulin target tissues improves resolution capacity and alleviates obesity-linked inflammation and insulin resistance in high-fat-fed mice. Diabetes 2010; 59: 3066-3073.

7 Smith BK, Holloway GP, Reza-Lopez S, Jeram SM, Kang JX, Ma DW. A decreased $n-6 / n-3$ ratio in the fat- 1 mouse is associated with improved glucose tolerance. Appl Physiol Nutr Metab 2010; 35: 699-706.

8 Guebre-Egziabher F, Rabasa-Lhoret R, Bonnet F, et al. Nutritional intervention to reduce the $n-6 / n-3$ fatty acid ratio increases adiponectin concentration and fatty acid oxidation in healthy subjects. Eur J Clin Nutr 2008; 62: 1287 1293.

9 Kris-Etherton PM, Harris WS, Appel LJ; American Heart Association. Nutrition Committee. Fish consumption, fish oil, omega-3 fatty acids, and cardiovascular disease. Circulation 2002; 106: 2747-2757.

10 Soriguer FJ, Tinahones FJ, Monzón A, et al. Varying incorporation of fatty acids into phospholipids from muscle, adipose and pancreatic exocrine tissues and thymocytes in adult rats fed with diets rich in different fatty acids. Eur $J$ Epidemiol 2000; 16: 585-594.

11 Pan DA, Storlien LH. Dietary lipid profile is a determinant of tissue phospholipid fatty acid composition and rate of weight gain in rats. J Nutr 1993; 123: $512-519$.

12 Field CJ, Ryan EA, Thomson AB, Clandinin MT. Diet fat composition alters membrane phospholipid composition, insulin binding, and glucose metabolism in adipocytes from control and diabetic animals. J Biol Chem 1990; 265: 11143-11150.

13 Fukuchi S, Hamaguchi K, Seike M, Himeno K, Sakata T, Yoshimatsu H. Role of fatty acid composition in the development of metabolic disorders in sucrose-induced obese rats. Exp Biol Med (Maywood) 2004; 229: 486-493.

14 Tsuji Y, Yamada K, Hosoya N, Moriuchi S. Digestion and absorption of sugars and sugar substitutes in rat small intestine. J Nutr Sci Vitaminol (Tokyo) 1986; 32: 93-100.

15 Kawai K, Okuda Y, Yamashita K. Changes in blood glucose and insulin after an oral palatinose administration in normal subjects. Endocrinol Jpn 1985; 32: 933-936.

16 Saito K, Lee S, Shiuchi T, et al. An enzymatic photometric assay for 2deoxyglucose uptake in insulin-responsive tissues and 3T3-L1 adipocytes. Anal Biochem 2011; 412: 9-17.

17 Bligh EG, Dyer WJ. A rapid method of total lipid extraction and purification. Can J Biochem Physiol 1959; 37: 911-917.

18 Ariyama H, Kono N, Matsuda S, Inoue T, Arai H. Decrease in membrane phospholipid unsaturation induces unfolded protein response. $J$ Biol Chem 2010; 285: 22027-22035.
Support Center for Advanced Medical Sciences, Institute of Health Biosciences, The University of Tokushima Graduate School for their technical assistance. This work was supported in part by Grants-in-Aid for Scientific Research (B) 22300237 and 22650180 from Japan Society for the Promotion of Science (JSPS), and from The Food Science Institute Foundation.

\section{Conflict of Interest}

M.K. and H.S. are employees of Meiji Co., Ltd. Other authors declare no conflict of interest associated with this manuscript.

19 Fu S, Yang L, Li P, et al. Aberrant lipid metabolism disrupts calcium homeostasis causing liver endoplasmic reticulum stress in obesity. Nature 2011; 473: $528-531$.

20 Ozcan U, Cao Q, Yilmaz E, et al. Endoplasmic reticulum stress links obesity, insulin action, and type 2 diabetes. Science 2004; 306: 457-461.

21 Ozcan U, Yilmaz E, Ozcan L, et al. Chemical chaperones reduce ER stress and restore glucose homeostasis in a mouse model of type 2 diabetes. Science 2006; 313: 1137-1140.

22 Shindou H, Shimizu T. Acyl-CoA: lysophospholipid acyltransferases. J Biol Chem 2009; 284: 1-5.

23 Abbott SK, Else PL, Hulbert AJ. Membrane fatty acid composition of rat skeletal muscle is most responsive to the balance of dietary $n-3$ and $n-6$ PUFA. Br J Nutr 2010; 103: 522-529.

24 Turk J, Gross RW, Ramanadham S. Amplification of insulin secretion by lipid messengers. Diabetes 1993; 42: 367-374.

25 Shirakawa J, Fujii H, Ohnuma K, et al. Diet-induced adipose tissue inflammation and liver steatosis are prevented by DPP-4 inhibition in diabetic mice. Diabetes 2011; 60: 1246-1257.

26 Tsintzas K, Norton L, Chokkalingam K, et al. Independent and combined effects of acute physiological hyperglycaemia and hyperinsulinaemia on metabolic gene expression in human skeletal muscle. Clin Sci (Lond) 2013; 124: 675-684.

27 Opara EC, Hubbard VS, Burch WM, Akwari OE. Characterization of the insulinotropic potency of polyunsaturated fatty acids. Endocrinology 1992; 130: 657-662.

28 Feng DD, Luo Z, Roh SG, et al. Reduction in voltage-gated $\mathrm{K}+$ currents in primary cultured rat pancreatic beta-cells by linoleic acids. Endocrinology 2006; 147: 674-682.

29 Ahrén B, Magrum LJ, Havel PJ, et al. Augmented insulinotropic action of arachidonic acid through the lipoxygenase pathway in the obese Zucker rat. Obes Res 2000; 8: 475-480.

30 Persaud SJ, Muller D, Belin VD, et al. The role of arachidonic acid and its metabolites in insulin secretion from human islets of langerhans. Diabetes 2007; 56: 197-203.

31 Funai K, Song H, Yin L, et al. Muscle lipogenesis balances insulin sensitivity and strength through calcium signaling. J Clin Invest 2013; 123: 1229-1240.

32 Rong X, Albert CJ, Hong C, et al. LXRs regulate ER stress and inflammation through dynamic modulation of membrane phospholipid composition. Cell Metab 2013; 18: 685-697.

33 Nugent C, Prins JB, Whitehead JP, Wentworth JM, Chatterjee VK, O'Rahilly $\mathrm{S}$. Arachidonic acid stimulates glucose uptake in 3T3-L1 adipocytes by increasing GLUT1 and GLUT4 levels at the plasma membrane. Evidence for involvement of lipoxygenase metabolites and peroxisome proliferatoractivated receptor gamma. J Biol Chem 2001; 276: 9149-9157.

34 Hii CS, Moghadammi N, Dunbar A, Ferrante A. Activation of the phosphatidylinositol 3-kinase-Akt/protein kinase B signaling pathway in arachidonic acid-stimulated human myeloid and endothelial cells: involvement of the ErbB receptor family. J Biol Chem 2001; 276: 27246-27255.

35 Hughes-Fulford M, Li CF, Boonyaratanakornkit J, Sayyah S. Arachidonic acid activates phosphatidylinositol 3-kinase signaling and induces gene expression in prostate cancer. Cancer Res 2006; 66: 1427-1433.

36 Covey TM, Edes K, Fitzpatrick FA. Akt activation by arachidonic acid metabolism occurs via oxidation and inactivation of PTEN tumor suppressor. Oncogene 2007; 26: 5784-5792.

37 Sato K, Arai H, Miyazawa Y, et al. Palatinose and oleic acid act together to prevent pancreatic islet disruption in nondiabetic obese Zucker rats. $J$ Med 
Invest 2008; 55: 183-195.

38 Tanaka T, Hidaka S, Masuzaki H, et al. Skeletal muscle AMP-activated protein kinase phosphorylation parallels metabolic phenotype in leptin transgenic mice under dietary modification. Diabetes 2005; 54: 2365-2374.

39 Suzuki A, Okamoto S, Lee S, Saito K, Shiuchi T, Minokoshi Y. Leptin stimulates fatty acid oxidation and peroxisome proliferator-activated receptor alpha gene expression in mouse $\mathrm{C} 2 \mathrm{C} 12$ myoblasts by changing the subcellular localization of the alpha2 form of AMP-activated protein kinase. $\mathrm{Mol}$
Cell Biol 2007; 27: 4317-4327.

40 Lee Y, Ravazzola M, Park BH, Bashmakov YK, Orci L, Unger RH. Metabolic mechanisms of failure of intraportally transplanted pancreatic beta-cells in rats: role of lipotoxicity and prevention by leptin. Diabetes 2007; 56: 2295 2301.

41 Ebihara K, Kusakabe T, Hirata M, et al. Efficacy and safety of leptinreplacement therapy and possible mechanisms of leptin actions in patients with generalized lipodystrophy. J Clin Endocrinol Metab 2007; 92: 532-541. 